\title{
Nasopharyngeal carcinoma presenting with rapidly progressive severe visual disturbance: a case report
}

\author{
Yoshinobu Kamio ${ }^{1}$, Naoto Sakai ${ }^{1 *}$, Goro Takahashi², Satoshi Baba ${ }^{3}$ and Hiroki Namba ${ }^{1}$
}

\begin{abstract}
Introduction: Nasopharyngeal carcinoma is one of the most difficult tumors to diagnose correctly at the initial phase because of the occasional lack of nasal symptoms. The perineural spread of the trigeminal nerve is one of the most common and important routes in the intracranial paracavernous extension of nasopharyngeal carcinoma, but visual loss is very rare.

Case presentation: We report the case of a 54-year-old Japanese man with nasopharyngeal carcinoma, who presented with rapid and severe disturbance of left monocular visual acuity and eye movement with a 10-month history of ipsilateral otitis media and facial pain. Magnetic resonance imaging revealed a lesion in the left fossa of Rosenmüller, pterygopalatine fossa, sphenoid and ethmoid sinus, and the left cavernous sinus extending to the orbital apex through the superior orbital fissure. The histopathological diagnosis was nonkeratinizing undifferentiated nasopharyngeal carcinoma. Epstein-Barr virus was detected by in situ hybridization. Although focal radiotherapy induced remarkable tumor shrinkage and relieved ocular motor disturbance and facial pain, his visual acuity did not improve.
\end{abstract}

Conclusion: The awareness of cranial nerves in addition to intracranial and orbital apex involvement, as in this case, is important for appropriate diagnosis and treatment planning of nasopharyngeal carcinoma.

Keywords: Cranial nerves, Epstein-Barr virus, Nasopharyngeal carcinoma, Orbital apex, Visual disturbance

\section{Introduction}

Nasopharyngeal carcinoma (NPC) is a relatively uncommon cancer with an incidence of 0.29 per 100,000 people per year in Japan [1]. However, there are certain populations in whom the incidence is considerably higher, including native and foreign-born Chinese, Southeast Asians, North Africans, and native people of the Arctic region [2]. The 2005 classification of the World Health Organization divides NPC on a pathological basis into three histological subtypes: keratinizing squamous cell carcinoma; nonkeratinizing carcinoma, which can be further divided into differentiated and undifferentiated subtypes; and basaloid squamous cell carcinoma. Undifferentiated nonkeratinizing NPC is the most common type of NPC and is strongly associated

\footnotetext{
* Correspondence: nsakaineurosurg@gmail.com

${ }^{1}$ Department of Neurosurgery, Hamamatsu University School of Medicine,

Handayama 1-20-1, Higashiku, Hamamatsu, Shizuoka 431-3192, Japan

Full list of author information is available at the end of the article
}

with the Epstein-Barr virus (EBV) in practically $100 \%$ of cases. The most common site of origin for NPC is the lateral wall of the nasopharynx, especially the fossa of Rosenmüller, followed by the superior posterior wall. Nearly half of patients complain of nasal symptoms. Symptoms related to Eustachian tube obstruction, such as serous otitis media, also commonly occur. Headache and cranial nerve involvement are features of more advanced stages. A direct lateral spread of NPC involves the third division of the trigeminal nerve (V3). The perineural spread of the $\mathrm{V} 3$ is one of the most common and important routes in the intracranial paracavernous extension of NPC [3], but visual loss is very rare [4-6].

We report here the case of a Japanese man with undifferentiated nonkeratinizing NPC, who presented with acute visual loss following facial pain and serous otitis media. We discuss the clinical features and diagnosis of this rare clinical entity. 


\section{Case presentation}

A 54-year-old Japanese man was admitted to our neurosurgical department for the investigation and treatment of acute visual loss of his left eye. He had been treated for left serous otitis media and left facial pain mimicking trigeminal neuralgia for 10 months by the otolaryngologist of a local hospital. Neurological examination on admission showed left visual acuity of no light perception and left eye movements disturbed in all directions. Gadoliniumenhanced, fat-suppressed, T1-weighted magnetic resonance (MR) imaging revealed a lesion in the left fossa of Rosenmüller, pterygopalatine fossa, sphenoid and ethmoid sinus, and the left cavernous sinus extending to orbital apex through the superior orbital fissure (Figure 1). With a tentative diagnosis of malignant skull base tumor, an endoscopic tumor biopsy from nasopharyngeal tissue was performed, but failed because the nasopharyngeal tissue appeared normal and the tumor could not be identified. Subsequently, the intraorbital tumor was explored by performing a left frontotemporal craniotomy with an anterior clinoidectomy. There was no clear plane between the tumor and the extraocular muscles; therefore, an infiltration was suspected. A biopsy of the tumor was performed and an intraoperative diagnosis of a malignant tumor was obtained. Histopathological findings were characterized by small round blue cells that were immunohistochemically positive for Ep-CAM/Ber-EP4 (epithelial marker) and CD117/c-Kit. Ki-67 positive cells totaled more than $90 \%$. EBV was detected by EBVencoded ribonucleic acid in situ hybridization (EBERISH; Figure 2). Considering the MR imaging appearance, the clinical course, and the histopathological findings, the patient was diagnosed with nonkeratinizing undifferentiated NPC, which originally occurred in the fossa of Rosenmüller and was perineurally invading along the V3 to the cavernous sinus.

He received focal radiotherapy at a dose of $46 \mathrm{~Gy}$ in 23 fractions, which remarkably reduced the tumor size and relieved left facial pain, facial palsy, facial hypoesthesia, ocular motor disturbance, hearing loss, and hypoglossal palsy. However, his left visual acuity did not improve. After the radiation therapy, ${ }^{18} \mathrm{~F}$-fluoro-2-deoxy-D-glucose positron emission tomography (FDG-PET) was negative. Therefore, adjuvant chemotherapy was not strongly advised and was eventually suspended. However, cervical lymph node metastases were detected by a
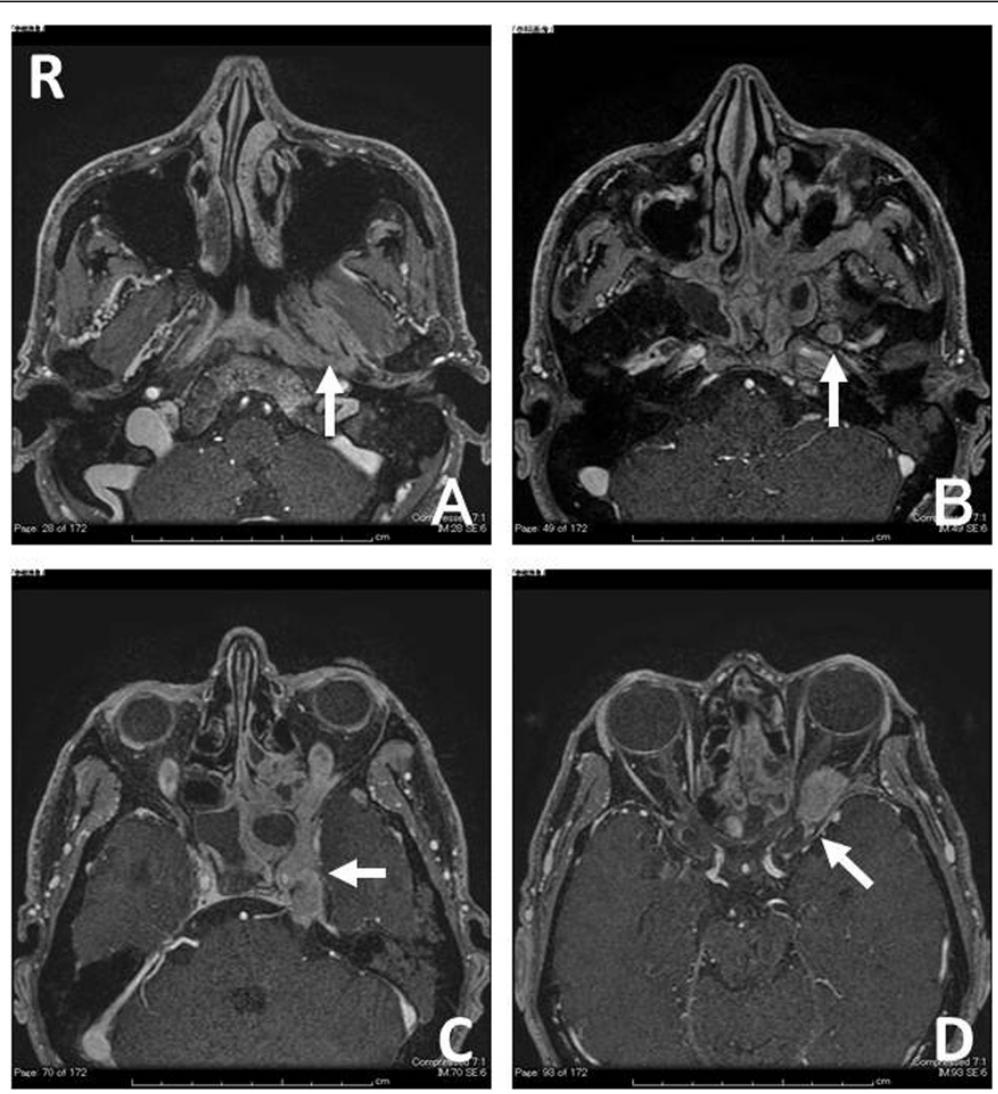

Figure 1 T1-weighted magnetic resonance imaging with gadolinium-diethylenetriaminepentaacetic acid showing a well-enhanced lesion in the fossa of Rosenmüller ( $A$; arrowhead, $R$; right), the foramen ovale (B; arrowhead), and the left cavernous sinus ( $C$; arrowhead) extending to orbital apex through the superior orbital fissure (D; arrowhead). 


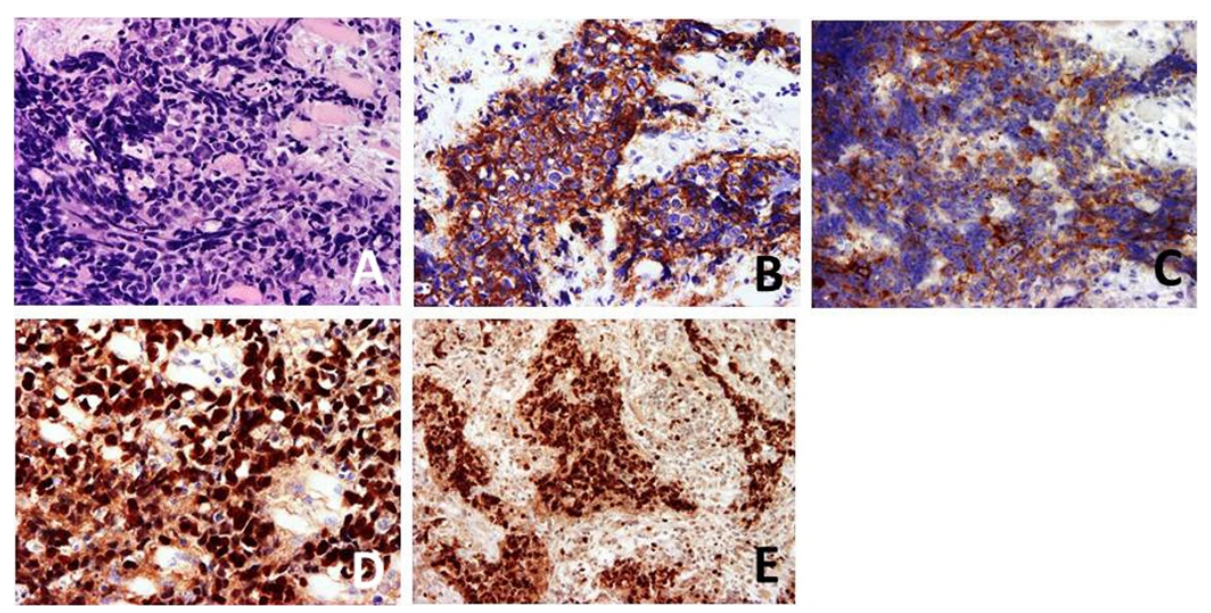

Figure 2 Hematoxylin-eosin staining (A) and immunohistochemical staining for Ep-CAM/Ber-EP4 (B), CD117/c-Kit (C), Epstein-Barr virus-encoded ribonucleic acid in-situ hybridization (D), and Ki-67 ( $\times 400$ magnification) (E).

repeat FDG-PET scan 8 months later. The patient underwent cervical lymphadenectomy followed by chemoradiation therapy. Although three cycles of cisplatin were scheduled, only one cycle could be completed because of leukopenia. He then received additional radiation therapy (60Gy/30 fractions/43 days). There was no recurrence 18 months after the initial treatment. However, left temporal and frontal lobe dural metastasis and lung metastasis were detected by repeat MR imaging and FDG-PET. Subsequently, he has received stereotactic radiotherapy and adjuvant chemotherapy with docetaxel hydrate, cisplatin, and fluorouracil. At present, 39 months after the initial treatment, he is still able to perform his daily activities (Karnofsky Performance Scale 80\%).

\section{Discussion}

The frequency of cranial nerve paralysis in NPC has a range of 8.0 to $12.4 \%$ [7]; cranial nerve invasion is a well-known poor prognostic factor in NPC [8-10]. Among the cranial nerves, the trigeminal nerve is most commonly involved in the extension of NPC. However, visual loss is very rare as an initial cranial nerve symptom of NPC [4-6]. NPC can also invade the inferior orbital fissure through the ethmoid or sphenoid sinus, as well as the orbita through the pterygopalatine fossa [11]. Although the incidence of trigeminal nerve involvement on MR imaging is high, it is often asymptomatic in NPC [3]. Su and Lui reported that patients could tolerate perineural infiltration of the extracranial segment of the trigeminal nerve by NPC in the early stage of the disease when the tumor is still confined to beneath the base of the skull [12].

In the present case, the patient was treated for left serous otitis media and left facial pain mimicking trigeminal neuralgia for 10 months before the acute visual loss.
These symptoms indicate that the tumor originally occurred at the fossa of Rosenmüller, then spread along the V3 and invaded the cavernous sinus and the intraorbita. Although he had serous otitis media and facial pain 10 months before the orbital symptoms, the main apparent cranial nerve paralysis in this case was left optic nerve paralysis with acute visual loss. Furthermore, there was no actual evidence of the cancer until the orbital symptoms occurred. We found only four cases in three case reports of initial optic nerve presentation of NPC [4-6], in which all patients presented with a rapid and progressive disturbance of visual acuity with poor prognosis of visual functions. All four patients had optic neuropathy (three monocular and one binocular). Histopathological subtypes of these cases were one keratinizing squamous cell carcinoma, two nonkeratinizing differentiated carcinoma, and one nonkeratinizing undifferentiated carcinoma.

Gadolinium-enhanced, fat-suppressed, T1-weighted MR imaging is very useful in detecting perineural tumor invasion and extracranial lesions [3]. In the present case, the MR image showing a lesion in the left cavernous sinus initially puzzled us regarding the primary site of the tumor. Although the imaging studies should include whole neck region in endemic areas in such a case, we initially performed the standard cranial MR imaging. We retrospectively examined the plain computed tomography $(\mathrm{CT})$ scan of his head and neck performed by the previous physician in the screening process, and noticed a slight enlargement of the left fossa of Rosenmüller. The fossa of Rosenmüller should be carefully observed on initial CT or MR imaging because it is the most common site of origin for NPC.

In the present case, we performed an endoscopic tumor biopsy from nasopharyngeal tissue with a 
tentative diagnosis of malignant skull base tumor, but could not reach the pathologic diagnosis. Although we subsequently performed open biopsy by craniotomy, a transnasal endoscopic biopsy could have easily and less invasively been performed instead of craniotomy [13].

In our patient, EBV was detected by EBER-ISH. The association of EBV is well established in nonkeratinizing NPC, particularly the undifferentiated subtype, detected in virtually all cases irrespective of the geographic origin. It seems reasonable to assume that EBV infection somehow contributes to the pathogenesis of nonkeratinizing NPCs [2]. Recently, cell-free circulating EBV DNA was detected in plasma and serum from patients with NPC and was reported as an independent prognostic maker for NPC. In endemic areas, an elevation of the plasma EBV DNA is one of the strong indicators of NPC [2,14]. By contrast, in EBV-negative squamous cell NPCs, other factors such as smoking and/or infection with human papillomaviruses might be involved in the pathogenesis [2]. In the present case, CD117/c-Kit was positive. C-Kit is a transmembrane growth factor receptor for stem cell factor that is not expressed in normal nasopharyngeal epithelial cells [15]. C-Kit protein was expressed in onethird of NPCs, restricted to cases of EBV-positive undifferentiated or nonkeratinizing carcinoma. Although patients with c-Kit expression are not significantly better survivors than those without, the molecular target therapy, imatinib mesylate $\left(\right.$ Gleevec $\left.^{\circ}\right)$, is potentially a new therapeutic approach in NPCs positive for c-Kit [15].

At present, radiation therapy is the first choice for $\mathrm{NPC}$, and cisplatin therapy is also thought to be effective $[2,8]$. The addition of cisplatin-based induction chemotherapy to radiation therapy was associated with a modest but significant decrease in relapse, and contributed to an improvement in disease-specific survival in advanced-stage NPC. However, there was no improvement in overall survival [2]. We initially did not administer chemotherapy to our patient after radiation therapy because locoregional metastasis was not detected by FDG-PET. However, cervical lymph node metastases, lung metastasis, and intracranial dural metastasis were observed during the follow-up period. Eventually the patient underwent cervical lymphadenectomy and received chemoradiation therapy. The initial treatment for the present case (focal radiation therapy: $46 \mathrm{~Gy} / 23$ fractions alone without chemotherapy and neck irradiation) was inadequate. In radiotherapy, a dose of 65 to $75 \mathrm{~Gy}$ should have been given to the primary tumor and 65 to $70 \mathrm{~Gy}$ to the involved neck nodes because the distant failure rate is relatively high $[2,7]$.

Although his left vision has not been recovered, he still lives independently. This clinical course suggests that we should have performed early nasal endoscopic biopsy and initiated adjuvant chemotherapy immediately after the initial high-dose radiation therapy, with continuing administration to control tumor recurrence.

\section{Conclusions}

NPC is one of the most easily misdiagnosed tumors because it does not present with nasal symptoms; rather, the patient may initially have unspecific signs and symptoms such as headache, hearing loss, and facial pain. Although symptoms often appear after intracranial invasion, early optic nerve involvement is very rare. If NPC is suspected, a rapid pathological diagnosis and a high-dose radiation therapy with early adjuvant chemoradiotherapy are essential to ensure prolonged patient survival.

\section{Consent}

Written informed consent was obtained from the patient for publication of this case report and any accompanying images. A copy of the written consent is available for review by the Editor-in-Chief of this journal.

\section{Abbreviations}

CT: Computed tomography; EBER-ISH: EBV-encoded ribonucleic acid in situ hybridization; EBV: Epstein-Barr virus; FDG-PET: ${ }^{18}$ F-fluoro-2-deoxy-D-glucose positron emission tomography; MR: Magnetic resonance; NPC: Nasopharyngeal carcinoma; V3: The third division of the trigeminal nerve.

\section{Competing interests}

The authors declare that they have no competing interests.

\section{Authors' contributions}

YK and NS devised the study design and concept, obtained the data and figures, drafted the manuscript and references, and carried out a critical review. GT, SB and HN reviewed the manuscript and added comments for discussion. All authors read and approved the final manuscript.

\section{Acknowledgement}

The authors thank Mrs M. Takahashi for her help in the preparation of this paper.

\section{Author details}

${ }^{1}$ Department of Neurosurgery, Hamamatsu University School of Medicine, Handayama 1-20-1, Higashiku, Hamamatsu, Shizuoka 431-3192, Japan. ${ }^{2}$ Department of Otolaryngology, Division of Head and Neck Surgery, Hamamatsu University School of Medicine, Handayama 1-20-1, Higashiku, Hamamatsu 431-3192, Japan. ${ }^{3}$ Department of Diagnostic Pathology, Hamamatsu University School of Medicine, Handayama 1-20-1, Higashiku, Hamamatsu 431-3192, Japan.

Received: 3 July 2014 Accepted: 8 September 2014 Published: 6 November 2014

\section{References}

1. Kimura Y, Suzuki D, Tokunaga T, Takabayashi T, Yamada T, Wakisaka N, Yoshizaki T, Murata H, Miwa K, Shoujaku H, Watanabe Y, Yamada N, Ito Y, Yuta A, Takeuchi K, Hosokawa S, Mineta H, Hasegawa Y, Fujimoto Y, Nakashima T, Fujieda S: Epidemiological analysis of nasopharyngeal carcinoma in the central region of Japan during the period from 1996 to 2005. Auris Nasus Larynx 2011, 38:244-249.

2. Wei WI, Sham JS: Nasopharyngeal carcinoma. Lancet 2005, 365:2041-2054.

3. Cui C, Lio L, Ma J, Liang S, Tian L, Tang L: Trigeminal nerve palsy in nasopharyngeal carcinoma: correlation between clinical findings and magnetic resonance imaging. Head Neck 2009, 31:822-828.

4. Park KA, Oh SY: Nasopharyngeal carcinoma presenting with rapidly progressive severe binocular optic neuropathy and periocular pain in a young man. J Neuroophthalmol 2010, 30:50-152. 
5. Bernardini FP, Croxatto JO, Orcioni GF, Bianchi S: Visual loss secondary to orbital apex invasion as the first manifestation of recurrent nasopharyngeal carcinoma. Ophthal Plast Reconstr Surg 2009, 25:248-250

6. Kao LY, Chuang HC, Liang YS: Visual loss as the initial presentation of nasopharyngeal carcinoma. J Clin Neuroophthalmol 1993, 13:24-26.

7. Cheng SH, Tsai SY, Horng CF, Yen KL, Jian JJ, Chan KY, Lin CY, Terng SD, Tsou MH, Chu N: A prognostic scoring system for locoregional control in nasopharyngeal carcinoma following conformal radiotherapy. Int I Radiat Oncol Biol Phys 2006, 66:992-1003.

8. Mo HY, Sun R, Sun J, Zhang Q, Hung WJ, Li YX, Yang J, Mai HQ: Prognostic value of pretreatment and recovery duration of cranial nerve palsy in nasopharyngeal carcinoma. Radiat Oncol 2012, 7:149.

9. Yap ML, Choo BA, Chan YH, Lu JJ, Mun LK, Tham IW: Outcomes following treatment for patients with cranial nerve involvement from nasopharyngeal cancer. J Med Imaging Radiat Oncol 2012, 56:548-553.

10. Liu L, Liang S, Li L, Mao Y, Tang L, Tian L, Liao X, Cui C, Lin A, Ma J: Prognostic impact of magnetic resonance imaging-detected cranial nerve involvement in nasopharyngeal carcinoma. Cancer 2009, 115:1995-2003.

11. Roh JL, Sung MW, Kim KH, Choi BY, Oh SH, Rhee CS, Ha JW: Nasopharyngeal carcinoma with skull base invasion: a necessity of staging subdivision. Am J Otolaryngol 2004, 25:26-32.

12. Su CY, Lui CC: Perineural invasion of the trigeminal nerve in patients with nasopharyngeal carcinoma. Imaging and clinical correlations. Cancer 1996, 78:2063-2069.

13. Tsirbas A, Kazim M, Close L: Endoscopic approach to orbital apex lesions. Ophthal Plast Reconstr Surg 2005, 21:271-275.

14. Wang WY, Twu CW, Chen HH, Jiang RS, Wu CT, Liang KL, Shin YT, Chen CC, Lin PJ, Liu YC, Lin JC: Long-term survival analysis of nasopharyngeal carcinoma by plasma Epstein-Barr virus DNA levels. Cancer 2013, 119:963-970.

15. Bar-Sela G, Kuten A, Ben-Eliezer S, Gov-Ari E, Ben-Izhak O: Expression of HER2 and C-KIT in nasopharyngeal carcinoma: implications for a new therapeutic approach. Mod Pathol 2003, 16:1035-1040

doi:10.1186/1752-1947-8-361

Cite this article as: Kamio et al:: Nasopharyngeal carcinoma presenting with rapidly progressive severe visual disturbance: a case report. Journal of Medical Case Reports 2014 8:361.

\section{Submit your next manuscript to BioMed Central and take full advantage of:}

- Convenient online submission

- Thorough peer review

- No space constraints or color figure charges

- Immediate publication on acceptance

- Inclusion in PubMed, CAS, Scopus and Google Scholar

- Research which is freely available for redistribution 